\title{
Vesicular Glutamate Transporter 1 Is Required for Photoreceptor Synaptic Signaling But Not For Intrinsic Visual Functions
}

\author{
Juliette Johnson, ${ }^{1,2,5}$ Robert T. Fremeau Jr, ${ }^{2,4,5}$ Jacque L. Duncan, ${ }^{1}$ René C. Rentería, ${ }^{1,2,5}$ Haidong Yang, ${ }^{1}$ Zhaolin Hua, ${ }^{2,4,5}$ \\ Xiaorong Liu, ${ }^{1,2,5}$ Matthew M. LaVail,, ${ }^{1,3}$ Robert H. Edwards, ${ }^{2,4,5}$ and David R. Copenhagen ${ }^{1,2,5}$ \\ Departments of ${ }^{1}$ Ophthalmology, ${ }^{2}$ Physiology, ${ }^{3}$ Anatomy, and ${ }^{4}$ Neurology, and ${ }^{5}$ Program in Neurosciences, University of California School of Medicine, San \\ Francisco, California 94143
}

\begin{abstract}
Glutamatergic neurotransmission requires vesicular glutamate transporters (VGLUTs) to sequester glutamate into synaptic vesicles. Generally, VGLUT1 and VGLUT2 isoforms show complementary expression in the CNS and retina. However, little is known about whether isoform-specific expression serves distinct pathways and physiological functions. Here, by examining visual functions in VGLUT1-null mice, we demonstrate that visual signaling from photoreceptors to retinal output neurons requires VGLUT1. However, photoentrainment and pupillary light responses are preserved. We provide evidence that melanopsin-containing, intrinsically photosensitive retinal ganglion cells (RGCs), signaling via VGLUT2 pathways, support these non-image-forming functions. We conclude that VGLUT1 is essential for transmitting visual signals from photoreceptors to second- and third-order neurons, but VGLUT1 is not necessary for intrinsic visual functions. Furthermore, melanopsin and VGLUT2 expression in a subset of RGCs immediately after birth strongly supports the idea that intrinsic vision can function well before rod- and cone-mediated signaling has matured.
\end{abstract}

Key words: ERG; VEP; immunohistochemistry; photoentrainment; pupillary light response; multielectrode array

\section{Introduction}

High-acuity, image-forming vision is initiated by photoreceptors that form a mosaic of light detectors at the back of the eye. Visual signals are transmitted from photoreceptors via glutamatergic synapses (Copenhagen and Jahr, 1989). For vesicle-mediated transmission in the nervous system, glutamate must be sequestered into synaptic vesicles that fuse with the plasma membrane to release the neurotransmitter onto postsynaptic neurons. Vesicular glutamate transporters (VGLUTs) exclusively load glutamate into synaptic vesicles (Bellocchio et al., 2000; Takamori et al., 2000). Antibodies to VGLUT1 stain synaptic terminals of all photoreceptors and bipolar cells, the principal classes of glutamatergic neuron in retina (Johnson et al., 2003; Sherry et al., 2003). In rodents, VGLUT2 antibodies stain retinal ganglion cells (RGCs), the output neurons of the retina, and a small subset

Received Feb. 22, 2007; revised May 21, 2007; accepted May 23, 2007.

This work was supported primarily by grants from the National Institutes of Health (D.R.C., R.H.E., J.J., J.L.D.) Additional support was provided by a core grant from National Eye Institute and by That Man May See (D.R.C.), Research to Prevent Blindness (D.R.C., J.L.D., M.M.L.), Foundation Fighting Blindness (J.L.D., M.M.L.), Bernard A. Newcomb Macular Degeneration Fund (J.L.D.), and Knights Templar Eye Foundation (J.J., X.L.). We thank members of the Copenhagen and Krizaj laboratories and Dr. Russell Van Gelder for valuable discussions during the course of this study. We thank Drs. Rebecca Seal, Lawrence Tecott, Louis Ptacek, Quasar Padiath, and Andreas Kadavanich for help with the circadian entrainment studies; Ann Schreiber, Vincent Wu, Carolyn Graybeal, and lvy Hsieh for their technical assistance; Dr. Travis Porco for helpful guidance on statistical analysis; and Dr. Ignacio Provencio for the melanopsin antibody.

Correspondence should be addressed to Dr. Juliette Johnson, Department of Ophthalmology, University of California, San Francisco, School of Medicine, 10 Kirkham Street, Room K-140, San Francisco, CA 94143-0730. E-mail: johnsonj@vision.ucsf.edu.

DOI:10.1523/JNEUROSCI.0815-07.2007

Copyright $\odot 2007$ Society for Neuroscience $\quad$ 0270-6474/07/277245-11\$15.00/0
( $\sim 10 \%$ ) of cones (Sherry et al., 2003; Fyk-Kolodziej et al., 2004; Wässle et al., 2006). It would be predicted from immunoreactivity patterns that VGLUT1 is essential for transmission of visual signals from photoreceptors to RGCs. However, this has not been physiologically established. We measured light-evoked responses in VGLUT1-null mice to assess the role of VGLUT1 in mediating this synaptic transmission.

Complementary expression of VGLUT1 and VGLUT2 in the brain suggests that these isoforms serve distinct and separable functions, but little is known about which physiological functions depend on this differential expression (Fremeau et al., 2001). In retina, it is possible that the expression of VGLUT1 in photoreceptors and bipolar cells serve visual functions different from the VGLUT2-expressing RGCs. In the absence of photoreceptor function, it has been shown that light activation of intrinsically photosensitive RGCs (ipRGCs) can mediate so-called intrinsic visual functions that include pupillary light responses (PLRs) and photoentrainment (Berson et al., 2002; Hattar et al., 2002; Sekaran et al., 2005). Even if photoreceptor signaling does require VGLUT1, this intrinsic vision might be preserved in VGLUT1-null mice. VGLUT2 is expressed in RGCs (Sherry et al., 2003), which could support synaptic transmission to the brain nuclei serving these functions. However, it is not known whether ipRGCs express VGLUT2. Additionally, neurons in the suprachiasmatic nucleus (SCN) and the hypothalamus, major centers for control of photoentrainment, predominantly express VGLUT1 (Ziegler et al., 2002), raising the possibility that these functions require VGLUT1 expression. We studied VGLUT1-null mice to determine whether VGLUT1 is required for intrinsic vision. 
Photoreceptors begin to make synapses with second-order neurons at approximately postnatal day 5 (P5) (Fisher, 1979) in the mouse. VGLUT1 expression is not observed in photoreceptors until approximately age P7 (Johnson et al., 2003; Sherry et al., 2003). This sets the minimum age when photoreceptor-mediated visual signals could be transmitted via RGCs to the brain. In contrast, light-evoked upregulation of c-fos expression in the SCN (Sekaran et al., 2005) and ipRGC activity are seen immediately after birth (Tu et al., 2005). That the glutamatergic ipRGCs signal to the SCN before onset of VGLUT1 expression in retina suggests that VGLUT2 or VGLUT3 must be expressed in ipRGCs. We used immunostaining to establish which VGLUT isoform serves to mediate light-evoked ipRGC transmission to the SCN in neonatal animals.

\section{Materials and Methods}

Animals. VGLUT1 knock-out (KO) mice were generated as reported previously (Fremeau et al., 2004b). VGLUT1-null mice and their heterozygous and wild-type (WT) littermates were studied. P30 Long-Evans rats (Simonsen, Gilroy, CA) were used for the preembedding electron microscopy experiments. Animals were fed and housed under a $12 \mathrm{~h}$ light/dark schedule. All animal procedures were approved by the University of California, San Francisco (UCSF) Institutional Animal Care and Use Committee and conformed to the National Institutes of Health Guide for the Care and Use of Laboratory Animals, the Public Health Service Policy, and the Society for Neuroscience Policy on the Use of Animals in Neuroscience Research.

Tissue preparation for immunohistochemical experiments. Retinal tissue was prepared as described previously (Johnson et al., 2004). Briefly, the eye was removed, the cornea was cut open or removed, and the eye was immediately immersed in $4 \%(\mathrm{w} / \mathrm{v})$ paraformaldehyde (PFA) in $0.1 \mathrm{M}$ PBS. The eye was fixed in PFA in $0.1 \mathrm{M}$ PBS for 30-60 min at room temperature. Eyes were rinsed in PBS, $\mathrm{pH} 7.4$, cryoprotected in 10, 20, and 30\% sucrose-PBS solutions, embedded in ornithine carbamyl transferase (OCT; Tissue-Tek, Elkhart, IN), and frozen on dry ice. Sections of the retina were cut perpendicular to the vitreal surface with a cryostat at $14-16 \mu \mathrm{m}$, mounted onto Colorfrost/Plus slides (Fisher Scientific, Pittsburgh, PA), and stored frozen at $-20^{\circ} \mathrm{C}$ until used for immunostaining.

Antibodies. A rabbit polyclonal antibody against VGLUT1 (Robert Edwards, UCSF, San Francisco, CA) and a commercially available guinea pig VGLUT1 antibody (Millipore, Billerica, CA) were used. We used rabbit polyclonal antibodies for calbindin (Millipore) and calretinin (Millipore). We used mouse monoclonal antibodies for PKC (Santa Cruz Biotechnology, Santa Cruz, CA), SV-2 (synaptic vesicle protein 2; Developmental Studies Hybridoma Bank, Iowa City, IA), tyrosine hydroxylase (Millipore), GAD65/67 (Developmental Studies Hybridoma Bank), and glutamine synthetase (BD Biosciences, Franklin Lakes, NJ). We used guinea pig antibodies for VGAT (vesicular GABA transporter), VGLUT2, and VGLUT3 (Millipore). A rabbit polyclonal for melanopsin was a kind gift from Dr. Ignacio Provencio (University of Virginia, Charlottesville, VA).

Immunohistochemical procedures. Retinal tissue was prepared as described previously (Johnson et al., 2003). Briefly, cryostat sections were washed in $0.1 \mathrm{M}$ PBS, incubated $12-48 \mathrm{~h}$ in primary antibody with 0.5 or $1 \%$ Triton X-100 and $10 \%$ normal goat serum at $4^{\circ} \mathrm{C}$, and then washed in $0.1 \mathrm{~m}$ PBS. In some experiments, antibodies were diluted in a blocking solution containing $10 \%$ bovine serum albumin instead of normal goat serum. To visualize binding of the primary antibodies, sections were incubated in secondary antibody conjugated to Alexa Fluor 488 or 594 (diluted 1:500-1:1000 in blocking solution; Invitrogen, Carlsbad, CA) for $1-2 \mathrm{~h}$ at room temperature. Sections were coverslipped with Vectashield (Vector Laboratories, Burlingame, CA) to retard fading. Wholemount retina was incubated in primary antibody for $4-5 \mathrm{~d}$ and in secondary antibody for 2-3 d.

In situ hybridization. VGLUT2 full-length cDNA was used for in situ hybridization (Fremeau et al., 2001). Digoxigenin-labeled antisense T7 and sense T3 probes (Roche, Palo Alto, CA) were prepared and applied as described previously (Liu and Green, 2002). Peroxidase-conjugated anti- digoxigenin peroxidase (1:500; Roche) was used to detect hybridized cRNA probes. Signals were visualized using the tyramide signal amplification system (PerkinElmer, Wellesley, MA). In some cases, immunostaining was performed after in situ hybridization.

Detection and image processing. For confocal microscopy, images were captured using a Zeiss (Thornwood, NY) Pascal confocal microscope. Image scale was calibrated and, if necessary, brightness and contrast were adjusted to highlight specific immunolabeling. For double-labeling experiments, matching images were captured in the Alexa Fluor 488 or Alexa Fluor 594 channels, pseudocolored, and merged using Adobe Photoshop 7.0 software (Adobe Systems, Mountain View, CA). "Bleedthrough" between fluorescent channels on the confocal microscope was eliminated by adjusting laser power and detector sensitivity and by sequential imaging of the fluorescent channels.

Statistical analysis. Results are expressed as the mean \pm SE (SEM). ANOVA tests were performed using Prism (GraphPad Software, San Diego, CA) to compare multiple samples in one group, and Student's $t$ test was performed to compare paired samples. In some cases, the MannWhitney test was used for statistical analysis. VGLUT1-null mice had no measurable electroretinogram (ERG) dark- or light-adapted b-waves or visual-evoked potential (VEP) responses; therefore, these were not included in the statistics.

Preembedding electron microscopy. Rats were perfused with 2\% PFA and $1 \%$ glutaraldehyde in PBS [electron microscopy (EM) fixative]. Anterior segments of hemisected eyecups were immersed in EM fixative for $10 \mathrm{~min}$ at room temperature. Retinas was dissected out and fixed for $3 \mathrm{~h}$ at room temperature and then rinsed and washed in PBS, cut into thin longitudinal strips, and stored in PBS at $4^{\circ} \mathrm{C}$. Retinas were placed in $30 \%$ sucrose in PBS overnight at $4^{\circ} \mathrm{C}$ and frozen in 2-methylbutane/LN2 and slowly thawed in a cold block and processed for electron microscopy (Johnson et al., 2004). Retinas were washed with $0.1 \%$ bovine serum albumin/Tris saline and $0.05 \%$ Tween 20, pH 7.4 (BTS) and quenched with $3 \% \mathrm{H}_{2} \mathrm{O}_{2}$ in BTS. Retinas were washed in BTS and blocked with Vector ABC kit (Vector Laboratories) and incubated in VGLUT1 antibody overnight at $4^{\circ} \mathrm{C}$. After washing with BTS, a Vector linking antibody was added for $60 \mathrm{~min}$ followed by an $\mathrm{ABC}$ reagent for $60 \mathrm{~min}$. The retinas were washed and incubated in DAB (Sigma, St. Louis, MO) for $10 \mathrm{~min}$ and fixed in Karnovsky's fixative for $60 \mathrm{~min}$. The retinas were reduced in $2 \%$ aqueous $\mathrm{OsO}_{4}$ and $1.5 \%$ ferrocyanide for $60 \mathrm{~min}$, rinsed, and added to Kellenburger buffer ( $2 \%$ buffered with uranyl acetate) at $37^{\circ} \mathrm{C}$ for 30 min. The retinas were dehydrated and embedded in Eponate 812 (Ted Pella, Redding, CA) and processed for electron microscopy.

Electroretinograms. The methods used were as described previously (Duncan et al., 2003). Threshold criterion amplitudes were $20 \mu \mathrm{V}$ for scotopic b-waves and $10 \mu \mathrm{V}$ for photopic b-waves.

$V E P$ recordings. VEPs were recorded in mice after being dark-adapted overnight. Pupils were dilated, and mice were anesthetized as described above. Recordings were made using a silver wire electrode placed under the scalp overlying the visual cortex (Peachey et al., 1997), and reference and ground electrodes were placed subcutaneously in the nose and the tail, respectively. Flash stimuli ranging from -3.6 to $0.9 \log \mathrm{cd} / \mathrm{s} / \mathrm{m}^{-2}$ were presented in a Ganzfeld bowl using the UTAS-E 3000 Visual Diagnostic System (LKC Technologies, Gaithersburg, MD) in order of increasing luminance. Flashes were presented at a frequency of $1.1 \mathrm{~Hz}$, and 200 consecutive responses were averaged at each intensity.

Pupillary light-response measurement. Mice were dark adapted for $1 \mathrm{~h}$. Under dim red illumination, the dark-adapted, unanesthetized mice were placed in a rodent restrainer device and positioned in front of an infrared video camera attached to a video monitor and recorder (ElectroDiagnostic Imaging, Redwood City, CA). A blue ( $\sim 480 \mathrm{~nm}$ ) light stimulus was delivered to the contralateral eye and pupillary constriction was monitored under infrared light. The videos were imported into iMovie (Apple, Cupertino, CA) and then analyzed in NIH Image. The pupil diameter was measured once per second for $5 \mathrm{~s}$ before the light onset and for $30 \mathrm{~s}$ after the onset. For higher temporal resolution, the pupil diameter was measured once every $100 \mathrm{~ms}$ for the first $7 \mathrm{~s}$ of the PLR. For analysis of the higher temporal resolution PLR, we found the best fit to the data was a function that consisted of an absolute delay followed by a single exponential decay. Each function was optimized with a least- 

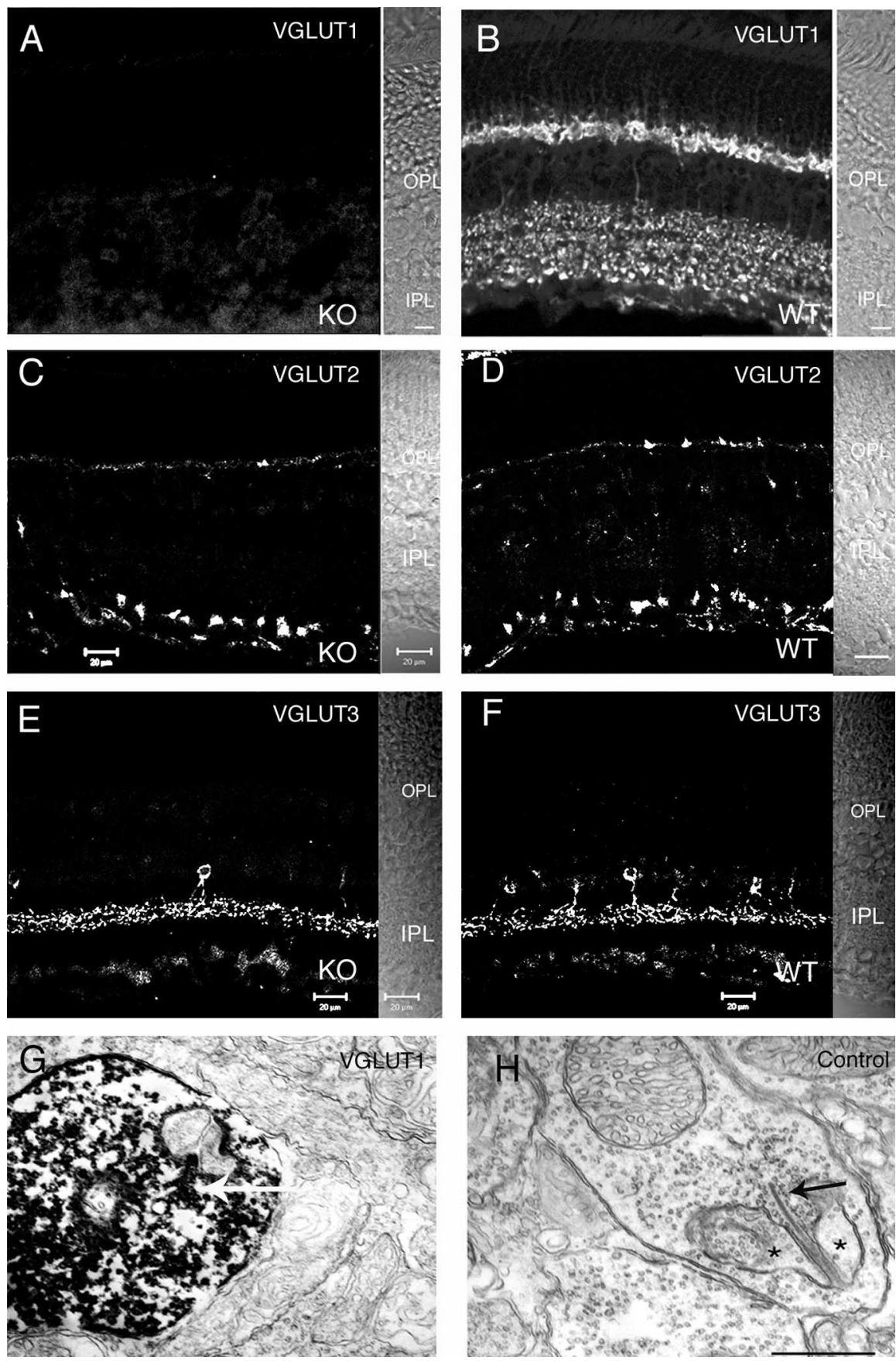

Figure 1. VGLUT1 is absent in VGLUT1-null mouse retina, whereas VGLUT2 and VGLUT3 are not upregulated. $\boldsymbol{A}$, No VGLUT1 immunostaining was observed in VGLUT1-null mouse retina. This demonstrates that the VGLUT1 antibody is specific for the VGLUT1 epitope and verifies complete deletion of VGLUT1 in the VGLUT1-null mouse. $\boldsymbol{B}$, VGLUT1 immunostaining was observed in the two synaptic layers of WT retina, the outer plexiform layer (OPL) and the inner plexiform layer (IPL). $\mathbf{C}-\boldsymbol{F}$, There was no difference in immunostaining between WT and VGLUT1-null retina for VGLUT2 or VGLUT3. Sections of mouse retina showed VGLUT2 immunostaining of retina ganglion cells and a small subset of cone photoreceptors in null (C) and VGLUT1 WT retina (D). VGLUT3 immunostained a subset of amacrine cells in null $(\boldsymbol{E})$ and WT retina $(\boldsymbol{F}) . \boldsymbol{G}, \boldsymbol{H}$, Preembedding immunohistochemistry and electron microscopy were used to examine VGLUT1 immunoreactivity in rod photoreceptor terminals in the rat retina. $\boldsymbol{G}$, Intense peroxidase reaction product labeled synaptic vesicles, including synaptic vesicles that were associated with ribbons (arrow). $\boldsymbol{H}$, No labeling of photoreceptor terminals was evident in tissue processed without VGLUT1 antibody. Asterisks indicate postsynaptic processes. Scale bars: $\boldsymbol{A}, \boldsymbol{B}, 10 \mu \mathrm{m} ; \boldsymbol{C}-\boldsymbol{F}, 20 \mu \mathrm{m} ; \boldsymbol{G}, \boldsymbol{H}, 500 \mathrm{~nm}$.

squares error-fitting routine ( $\mathrm{R}$ statistical package, http://www. r-project.org). To compare each data set, we took the time point when the fitted curves dropped by $10 \%$ from the maximum pupil dilation diameter (Lucas et al., 2001). These differences of the delays between WT and VGLUT1-null mice were statistically analyzed using the Student's or Welch's $t$ test. Heterozygote responses were indistinguishable from WT; thus, we have pooled this data and refer to it as WT.

Multielectrode array extracellular recording from retina. Multielectrode array data recordings of light-evoked ganglion cell spiking were acquired and analyzed as described previously (Tian and Copenhagen, 2003; Rentería et al., 2006). The very long latency, longer-duration light responses were categorized as originating from intrinsically photosensitive RGCs based on the similarity to ipRGC responses characterized by $\mathrm{Tu}$ et al. (2005). We confirmed the inability of synaptic blockers to eliminate the responses (see Fig. 4). Because in some cases, the response to the first flash of light would lead to sustained firing across several stimulus time periods, it was problematical to show stimulustriggered ipRGC responses averaged across the retina. Thus, for clarity, only the first light responses were used for the population histograms shown in the figures.

Analysis of ambulation activity. Mice were housed for at least 2 weeks in a $12 \mathrm{~h}$ light/dark cycle before the start of the experiment. For the ambulation experiments, the mice were housed individually in cages with bedding, food, and water. To assess activity, beam breaks were collected continuously over a $23 \mathrm{~h}$ period each day. (San Diego Instruments, San Diego, CA). Ambulation activity was sampled every $10 \mathrm{~min}$ (Ralph and Caine, 2005). Supplemental food was added to both the WT and null cages. The mice were placed in $12 \mathrm{~h}$ light/dark conditions for the first $5 \mathrm{~d}$ and then switched to constant darkness from day 6 until the completion of the experiment on day 13. Actograms and period analysis was generated using Clocklab (Actimetrics, Wilmette, IL). Heterozygote responses were indistinguishable from WT; thus, we have pooled these data and refer to it as WT.

\section{Results}

\section{VGLUT1 is absent in VGLUT1-null} mouse retina, and VGLUT2 and VGLUT3 are not upregulated

VGLUT1-null mice, generated as reported previously (Fremeau et al., 2004b), were studied. To verify that VGLUT1 expression was deleted in these VGLUT1-null mice, we immunostained retinas of null mice with VGLUT1 antibodies. VGLUT1 staining was absent in VGLUT1-null mice, whereas VGLUT1 antibodies in WT retina showed robust staining in the outer plexiform layer and inner plexiform layer, in which the photoreceptor and bipolar synaptic terminals respectively reside (Fig. $1 A-B$ ). Figure $1 G$ confirms by electron microscopy that VGLUT1 antibodies are localized to synaptic vesicles in ribbon synapses similar to localization of VGLUT1 to vesicles in the CNS (Bellocchio et al., 1998). No change in the immunoreactivity patterns of VGLUT2 or VGLUT3 was detected in the retinas of VGLUT1-null mice (Fig. 1C-F). This indicates that there is not a compensatory upregulation of either of these two VGLUT isoforms in the absence of VGLUT1 expression. 
ERG recordings show that no photoreceptor-driven visual signals are transmitted to $\mathrm{ON}$ bipolar cells in VGLUT1-null mice

Dark-adapted ERG

We tested how deletion of VGLUT1 affected synaptic transmission from rod and cone photoreceptors. Consistent with intense expression of VGLUT1 in photoreceptor terminals of WT mice, ERG recordings indicated that VGLUT1 deletion eliminated rod-driven visual transmission but had no effect on photoresponses of rods. Figure 2, $A$ and $B$, shows representative dark-adapted single-flash ERG responses from WT and VGLUT1-null littermates. The dark-adapted a-wave represents the light-evoked mass activity of rod photoreceptor outer segments, whereas the dark-adapted b-wave reflects light-evoked summed responses of roddriven ON bipolar cells (Robson and Frishman, 1995). In Figure $2 \mathrm{~A}$, upward going $b$-waves can be observed at all light intensities more than $-4.6 \mathrm{~cd} \mathrm{~s} / \mathrm{m}^{2}$. Higher-light-intensity flashes evoked a-waves and b-waves that become larger and faster with higher stimulus intensities. In VGLUT1-null retinas, in contrast, no b-waves were observed at any stimulus intensity (Fig. 2 $B, C$ ). The amplitude and implicit time (i.e., time to peak) of darkadapted a-waves were indistinguishable in VGLUT1-null and WT mice [Fig. 2C, a-wave amplitude $(p>0.05, n=11 / 12 \mathrm{WT} / \mathrm{KO}$, ANOVA); a-wave implicit time $(p>0.05, n=11 / 8 \mathrm{WT} / \mathrm{KO}, \mathrm{ANOVA})]$. These results indicate that intrinsic rod photoresponsiveness is normal in VGLUT1null mice, whereas synaptic transmission to rod-driven bipolar cells is abolished.

\section{Light-adapted ERG}

To evaluate the effect of VGLUT1 deletion on cone-driven retinal function, we investigated light-adapted ERG responses in VGLUT1-null mice. Cone signaling is assessed by presenting flashes during background illumination. The light-adapted a-wave is negligible in the mouse (Peachey et al., 1993); therefore, we searched for a light-adapted b-wave. Figure 2, $D$ and $E$, shows representative single-flash, light-adapted, full-field ERG responses from WT and VGLUT1-null mice. In WT mice, the lightadapted ERG showed a prominent b-wave (Fig. 2D,F). In contrast, no b-wave was present in VGLUT1-null mice at any light intensity in the light-adapted retina (Fig. 2.E,F).

In summary, our ERG recordings indicate that absence of VGLUT1 results in abolishment of visually evoked synaptic transmission from rod and cone photoreceptors to the postsynaptic ON bipolar cells.

Retinal ganglion cell and visual-evoked potential recordings from cortex show no short-latency, photoreceptor-mediated light responses

RGCs are postsynaptic to bipolar cells. Recordings from RGCs provide a more sensitive and higher gain method to detect visual signals from rods and cones than ERGs and show light-evoked activity in both the ON and OFF pathways (Copenhagen et al., 1990; Tian and Copenhagen, 2003). Typical short-latency ONOFF responses, recorded from RGCs in a P33 WT retina are shown in Figure $3 A$. The right trace shows a typical response from RGCs in a VGLUT1-null retina (Fig. 3B). No short-latency ON or OFF responses were recorded at any age in VGLUT1-null retina.

Visual-evoked potentials are in vivo light-evoked responses that can be recorded from the mammalian visual cortex and reflect visual responses in the cone pathways. To examine further whether the cone pathway remained functional in VGLUT1-null mice but might have been missed in the in vitro multielectrode array (MEA) and the ERG recordings, we measured VEPs in WT and VGLUT1-null mice. Figure 3, $C$ and $D$, shows representative VEP responses to the same light intensities recorded from a WT and a VGLUT1-null mouse. The VEP waveform in Figure $3 C$ has the characteristic shape of WT mice (Peachey et al., 1997). Figure $3 D$ shows that no visually evoked response could be detected in VGLUT1-null mice at any stimulus intensity. WT mice had an average implicit time of $68.6 \pm 10.8 \mathrm{~ms}$. VEPs were similar in WT and VGLUT1 het mice. This was assessed by the similarity of the implicit times of the negative-going N1 component, a commonly used parameter to compare VEPs in different animals (Peachey et al., 1997). Our VEP recordings indicate that light-evoked visual responses are absent from the visual cortex of VGLUT1-null mice (Fig. 3D). Although we did observe a fast, small wave in the VEP for VGLUT1-null mice (Fig. 3D, middle and bottom), which was not present in the WT, this wave was not correlated with the light stimuli because it remained when the light path was physically blocked (Fig. 3D, middle and bottom, gray line). It was associated with the sound of the shutter driving the light stimulus and likely 


\section{MEA}

$$
\text { A }
$$$$
\text { WT }
$$
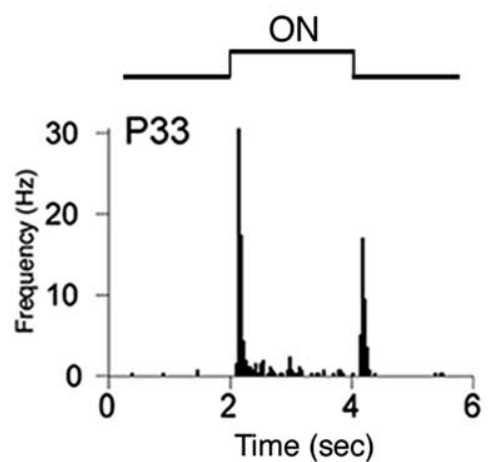

\section{VEP}

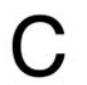

WT

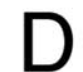

$\mathrm{KO}$

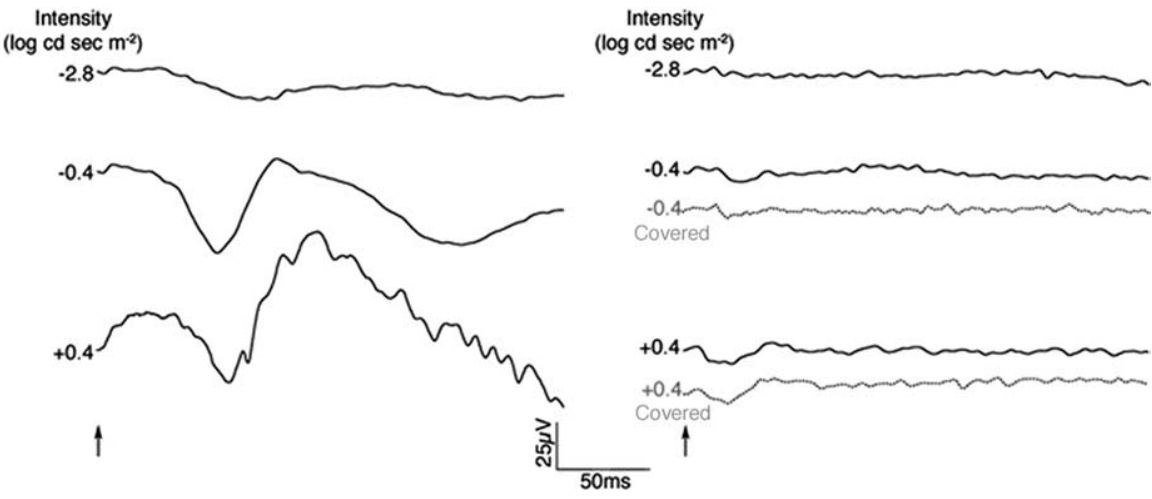

Figure 3. Retinal ganglion cell and visual-evoked potential recordings show no short-latency, rod- or cone-mediated light responses. Responses to a large-field light stimulus from multiple RGCs using an MEA. The average peristimulus time histograms of the first light-response trial to a 2 s light presentation are shown. $\boldsymbol{A}, \boldsymbol{B}, \mathrm{RGCs}$ in WT $(\boldsymbol{A})$ or VGLUT1-null $(\boldsymbol{B})$ retinas were recorded simultaneously using a multielectrode array. $\boldsymbol{A}$, In adult WT retina, RGCs at age P33 $(\boldsymbol{A})$ had well defined, transient $0 \mathrm{~N}, 0 \mathrm{FF}$, or ON-OFF responses. In contrast, at P31, a VGLUT1-null retina $(\boldsymbol{B})$ had only spontaneous spiking; no light responses were recorded. $\boldsymbol{C}, \boldsymbol{D}$, Representative VEP responses from a WT and VGLUT1-null mouse. VEPs were abolished at all stimulus luminances in the VGLUT1-null mouse (D), indicating that retinal activity was not transmitted to the visual cortex. In the VGLUT1-null mouse, a small fast, downward wave was observed ( $\boldsymbol{D}$, middle and bottom), which was not found in WT. This wave persisted when the eyes were completely covered, indicating that it is not associated with light-evoked activity. Instead, this wave is likely an auditory response to the noise generated by the opening of the light source shutter. The gray traces show the experiment repeated with the eyes covered.

reflects a short-latency cortical response driven by the auditory system (Barth et al., 1995). Together, these data strongly support the conclusion that visual signals from rods and cones are not transmitted to output neurons of retina or to higher visual centers in VGLUT1-null mice.

Light-evoked responses of intrinsically photosensitive RGCs remain in VGLUT1-null mice

$\mathrm{Tu}$ et al. (2005) characterized long-latency light responses of ipRGCs in neonatal mice using a MEA. We found similarly responding RGCs in retinas of both WT and VGLUT1-null mice.
Figure $4 \mathrm{~A}$ (left) shows the average peristimulus histograms of light responses derived from 88 RGCs in a P11 WT mouse. Figure $4 A$ (right) shows a light-evoked histogram of spike activity from all 61 RGCs recorded from a P12 VGLUT1-null retina. A comparison of the number of ipRGCs recorded from WT and VGLUT1null retina in $\mathrm{P} 11-\mathrm{P} 13$ mice shows more ipRGCs recorded in the null mice. Overall, $32 \pm 12 \%$ of the RGCs detected by their spiking had ipRGC-like responses in WT mice ( 428 total cells recorded from $n=5$ retinas) compared with $81 \pm 6.3 \%$ in the VGLUT1-null mice (333 total cells recorded from $n=5$ retinas; $p<0.01$, Mann-Whitney).

To rule out the possibility that the longlatency light responses were driven synaptically by visual signals originating in rods and cones, we blocked synaptic pathways pharmacologically. Similar to the findings of Tu et al. (2005), we found that glutamatergic and nicotinic receptor antagonists (100 $\mu \mathrm{m}$ DL-AP5; $20 \mu \mathrm{m}$ CNQX; $10 \mathrm{~nm}$ epibatidine) did not abolish the light responses (Fig. $4 B$, left and right). We conclude that these responses, which exhibit long latencies and continue after stimulus offset, originate from the ipRGCs.

The density of ipRGCs declines rapidly a few days before eye opening in WT mouse (Sekaran et al., 2005). The decline in ipRGCs during development appeared maturationally delayed in VGLUT1-null mice. In the P14-P16 group of WT mice, only $4.1 \pm 1.8 \%$ of light-responsive RGCs showed ipRGC-like responses (374 total cells in $n=5$ retinas). In contrast, $51 \pm$ $7.4 \%$ of the spiking RGCs in young VGLUT1-null retina showed ipRGC-like responses ( 161 total cells from $n=2$ retinas). Figure $4 A$ (bottom) shows average peristimulus histograms from P14 WT and P16 VGLUT1 retinas, respectively.

\section{Intrinsic, non-image-forming vision is} functional in VGLUT1-null mice Pupillary light response

Although our ERG, MEA, and VEP data indicated that VGLUT1-null mice do not have photoreceptor-driven vision, our MEA recordings showed responses from ipRGCs that are known to drive non-image-forming visual functions. Consequently, we first tested whether a PLR was present in VGLUT1-null mice. We found that the pupils of both WT and VGLUT1-null mice responded to a bright light step, with a fast constriction of the pupil (Fig. $5 A, B$ ). Figure $5 B$ shows representative infrared photographs of PLRs in a WT and VGLUT1-null mouse in response to a bright $\left(2 \mathrm{~mW} / \mathrm{cm}^{2}\right)$ blue $(\sim 480 \mathrm{~nm})$ light stimulus. Measurement of the time course of pupil size (measured once per second) revealed that the PLR was preserved in the VGLUT1-null mouse (Fig. 5A,C). Statistical analysis of the 
A

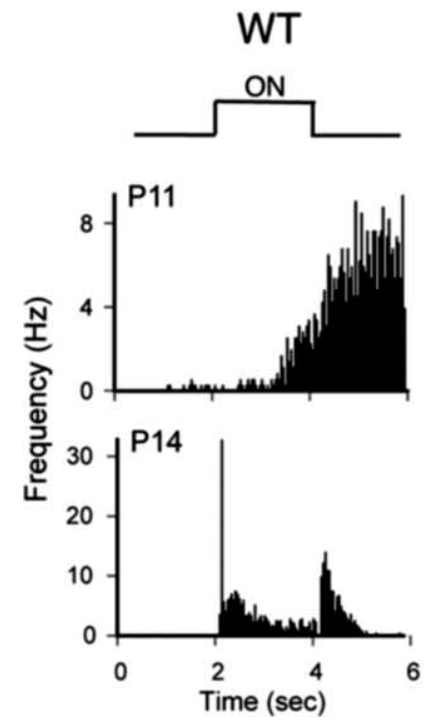

B

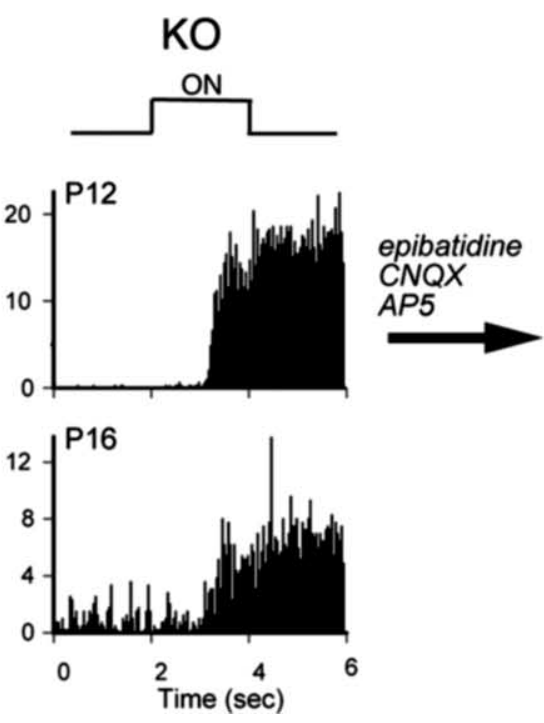

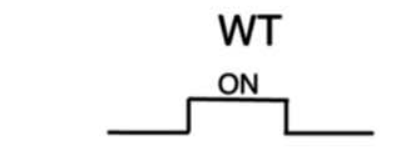
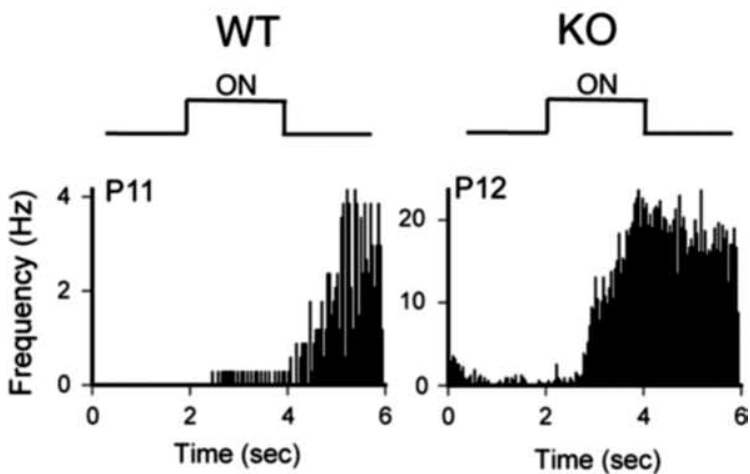

Figure 4. Intrinsic, non-image-forming vision is preserved in VGLUT1-null mice. Responses to a large-field light stimulus from multiple RGCs recorded with a multielectrode array. The average peristimulus time histograms of the first light-response trial to a 2 s light presentation. $A$, RGCs in WT (top left) or VGLUT1-null (top right) retinas were recorded simultaneously using a multielectrode array. At P11-P12, an age before eye-opening, RGCs recorded from WT (top left) and VGLUT1-null (top right) retina showed a similar sluggish sustained response characteristic of ipRGCs. After eye-opening, a P14 WT retina (bottom left) responded with typical rod- and cone-driven ON and OFF responses. These responses were absent in a P16 VGLUT1-null retina (bottom right), which instead showed ipRGC-like responses. $\boldsymbol{B}$, The responses of WT (left) and VGLUT1-null (right) RGCs at an age before eye-opening (P11 or P12) persisted in the presence of a drug mixture that blocked excitatory synaptic transmission.

plateau response, of $5-15 \mathrm{~s}$ after stimulus onset, revealed no significant difference ( $p>0.05, n=5 \mathrm{WT}$ and $n=4$ null, $t$ test).

Interestingly, higher-resolution measurement of the pupil diameter once every $100 \mathrm{~ms}$ during the first $7 \mathrm{~s}$ of the PLR revealed an initial delay in the VGLUT1 KO PLR compared with WT (Fig. 5B). We found the best fit to the data was a function that consisted of an absolute delay followed by a single exponential decay. Following Lucas et al. (2001), we calculated the time after stimulus onset when there was considerable pupil constriction $(10 \%$ from maximum diameter). The WT revealed this pupil constriction at $0.44 \mathrm{~s}$ $(0.44 \pm 0.05 \mathrm{~s}$, mean, SEM $)$, whereas the $\mathrm{KO}$ showed this constriction at $0.76 \mathrm{~s}$ $(0.76 \pm 0.12 \mathrm{~s}$, mean, SEM $)$. There was a significant delay of $\sim 0.3$ $s$ in the KO compared with the WT $(p<0.03, n=5 \mathrm{WT}$ and $n=$ 4 null, Student's $t$ test). The slower PLR in the VGLUT1 KO mouse is similar to a $0.3 \mathrm{~s}$ delay found in the rodless coneless mouse strain (Lucas et al., 2001). Taken as a whole, VGLUT1 expression is not required for the PLR.

\section{VGLUT1-null mice are photoentrainable}

The SCN is the site of the master circadian clock in mammals (Van Gelder, 2003). Photoentrainment of the clock results from light-level information transmitted from ipRGCs to the SCN. To test this aspect of the non-image-forming visual system, we determined whether VGLUT1-null mice can be photoentrained to a $12 \mathrm{~h}$ light/dark cycle. Previous studies demonstrated strong labeling for VGLUT1 mRNA in the SCN but no detectable VGLUT2 mRNA or immunostaining of cell bodies in this nucleus (Ziegler et al., 2002; Lin et al., 2003). These previous studies
B

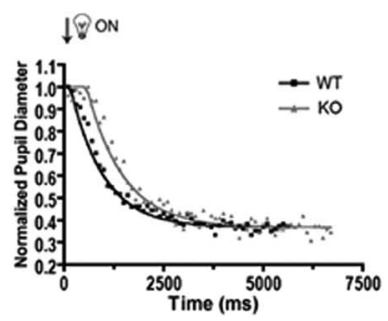

C

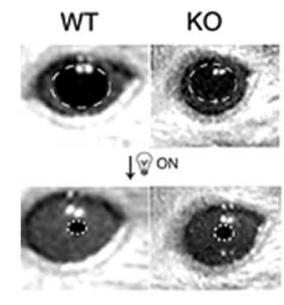

Figure 5. VGLUT1-null mice retain their intrinsic vision whereby they have pupillary light responses. $\boldsymbol{A}$, Mean normalized pupil diameter versus time in WT and VGLUT1-null mice $(n=5 / 4$ WT/KO). $B$, Representative higher temporal resolution time course

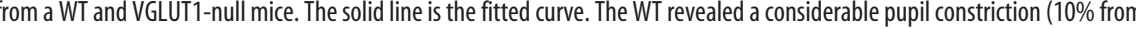
maximum diameter) at $0.44 \mathrm{~s}(0.44 \pm 0.05 \mathrm{~s}$, mean, SEM), whereas the KO showed this constriction at $0.76 \mathrm{~s}(0.76 \pm 0.12 \mathrm{~s}$ Student's $t$ test). C, Representative photos showing a pupillary light response in a WT (left) and VGLUT1-null (right) mouse. Dotted lines indicate circumference of the pupil.

predict that, even if the VGLUT1-null retina were able to signal a change in light via VGLUT2-mediated transmission, this signaling pathway could be disrupted because of a lack of VGLUT1 expression in the SCN output neurons (Ziegler et al., 2002; Lin et al., 2003).

However, as seen in Figure $6 A$, representative actograms for WT and VGLUT1-null mice show that both groups of mice are photoentrainable. The locomotor activity of the mice was assessed using photobeam breaks. The activity levels were collected in $10 \mathrm{~min}$ bins. The animals were kept in a $12 \mathrm{~h}$ light/dark cycle for the first $5 \mathrm{~d}$ and then switched to a dark/dark cycle from day 6 through day 13. The actograms indicated that the average period length in light/dark for the WT mice was $23.8 \mathrm{~h}$ (23.8 h for 4 of 4 $\mathrm{WT}$ ), and for the KO, it was $23.7 \pm 0.125$ (mean \pm SEM, $n=4$ ). The average period length in dark/dark for the WT mice was $23.4 \pm 0.22$, and for the KO, it was $23.7 \pm 0.1$ (mean $\pm \mathrm{SEM}, n=$ $5 \mathrm{WT}$ and $n=3 \mathrm{KO}$ ). We did not find a significant difference 

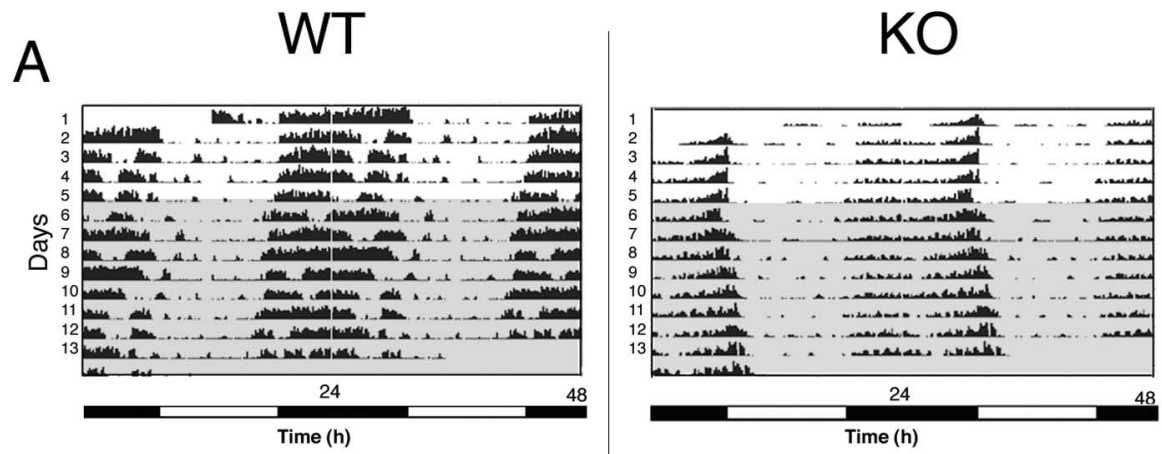

B

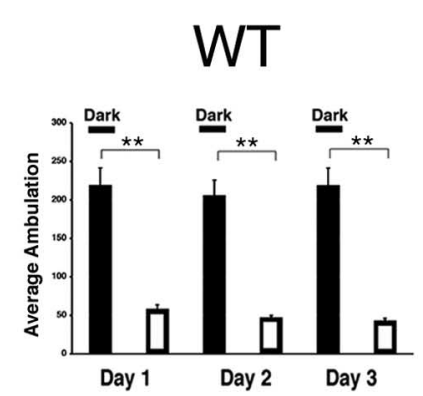

(Fig. 7C). In whole-mounted retinas, a subset of VGLUT2-immunostained ganglion cell bodies also had melanopsin immunoreactivity in both WT and VGLUT1null retina (Fig. $7 E-J$ ). More than $97 \%$ of the RGCs were found unequivocally to express VGLUT2 (data not shown). These findings are strong evidence that ipRGCs use VGLUT2 for transmitting visual signals from the ipRGCs.

Light induces activity in ipRGCs and c-fos expression in the suprachiasmatic nucleus of mice just after birth (Sekaran et al., 2005). Our VGLUT2 immunostaining data in young neonatal mice is consistent with the idea that glutamatergic signals from ipRGCs are mediated by VGLUT2 at these early ages, a period before photoreceptors are functional. We find that retinas of young postnatal mice express VGLUT2 (Sherry et al., 2003) with strong labeling of cell bodies apparent in P1, P5, and P8 WT retina (Fig. $8 A-C$ ). Immunostaining patterns of VGLUT2 in VGLUT1-null and WT retina were indistinguishable (Fig. $8 D-F)$. As in adults, VGLUT2 colocalized with melanopsin in RGCs throughout normal postnatal development [P3, P5, P7, P12, and P16 WT retina (Fig. 8G-U)]. VGLUT2 expression in these neurons is integral to the intrinsic visual system, and this early expression of VGLUT2 confers light-responsive functionality in very young neonates, before most retinal circuitry has developed. Because VGLUT3

between the VGLUT1 WT and KO light/dark or dark/dark period lengths. In addition, as seen in Figure $6 \mathrm{~B}$, both the WT and VGLUT1-null mice showed a significant increase in ambulation in the dark phase compared with the light phase, confirming that they can be photoentrained [Fig. $6 B$, WT ( $p<0.01, n=5, t$ test $)$; $\mathrm{KO}(p<0.05, n=4, t$ test $)]$.

\section{Melanopsin-containing RGCs express VGLUT2}

The above results demonstrate that VGLUT1 expression is not required in the retina or in the neuronal pathways of the pupillary or photoentrainment systems to mediate these intrinsic visual functions. Therefore, other VGLUT isoforms must be used by the ipRGCs to conduct glutamatergic signals from the retina to their target (suprachiasmatic and preoptic tectal nuclei) in the nonimage-forming visual brain areas. In the rat retina, VGLUT2 mRNA and protein have been localized to cell bodies in the ganglion cell layer (GCL) (Fujiyama et al., 2003), and retinal ganglion cells were found to be the source of VGLUT2-positive puncta in the LGN (Land et al., 2004). Although VGLUT3 immunoreactivity has been found in the somas of some cells in the GCL of the cat (Fyk-Kolodziej et al., 2004), in the mouse and rat retina, VGLUT3 immunostaining has only been found in a subset of amacrine cells with cell bodies in the inner nuclear layer (Haverkamp and Wässle, 2004; Johnson et al., 2004).

In situ hybridization in retinal slices, using VGLUT2 mRNA probes, showed strong labeling of cell bodies in the GCL (Fig. $7 \mathrm{~A}, C)$. Immunoreactivity for melanopsin, the pigment responsible for ipRGC light sensitivity (Berson et al., 2002) colocalized with a subset of these VGLUT2 mRNA-labeled ganglion cells expression is not found until P7-P8 in the mouse retina (Johnson et al., 2004), VGLUT2 becomes critical in mediating this early, non-image-forming vision.

\section{Discussion}

By examining visual functions in VGLUT1-null mice, we demonstrate that VGLUT1 is necessary for synaptic signaling of visual-evoked responses from photoreceptors. The absence of photoreceptor signaling in the VGLUT1-null mice allowed us to unmask the intrinsic visual system. The preservation of pupil responses and the ability to be photoentrained indicates that neurons expressing other VGLUT isoforms are sufficient to operate these intrinsic functions. Our identification of VGLUT2 expression in melanopsin-containing RGCs during early neonatal development leads us to conclude that intrinsic visual functions are possible before the maturational development of photoreceptormediated visual signaling.

\section{VGLUT1 expression is necessary for synaptic transmission of photoreceptor-driven visual responses}

In the retina, photoreceptor and bipolar cells are the principal glutamatergic neurons carrying high-acuity, imaging-forming visual signals to the RGCs, the retinal output neurons. VGLUT1 antibodies immunostain terminals of photoreceptors and bipolar cells (Johnson et al., 2003; Sherry et al., 2003). Using electron microscopy, we show here that this VGLUT1 immunoreactivity is localized to synaptic vesicles, consistent with the principal role of VGLUTs of loading glutamate into vesicles (Bellocchio et al., 
2000; Fremeau et al., 2004a). Our present study is the first physiological demonstration that VGLUT1 expression is required to transmit image-forming visual signals from photoreceptors to retinal output neurons (Figs. 2-4). Our results provide strong support for vesicle-mediated exocytosis being the mechanism for synaptically transmitting visual signals from photoreceptors (Schwartz, 1986).

Although VGLUT2 is considered the major isoform used in glutamatergic pathways from retina to brain (Fujiyama et al., 2003), we cannot conclude whether VGLUT1 neurons can mediate visual signaling in the thalamocortical pathways. Additional dual-recording experiments in retina and LGN or visual cortex would be needed to establish or rule out a role for VGLUT1.

Any visual signaling mediated by VGLUT2-expressing cones is below detection limits

A small subset of cones $(\sim 10 \%)$ coexpress VGLUT1 and VGLUT2 (Wässle et al., 2006). We found that there is no detectable difference in VGLUT2 staining between WT and VGLUT1-null retina (Fig. 1C,D), suggesting that there is no upregulation of VGLUT2 in the VGLUT1-null retina. It has been proposed that a positive-going wavelet following the a-wave in our scotopic ERG recordings (Wässle et al., 2006), which is observed at higher intensity scotopic flash responses (Fig. 2B), is attributable to b-waves generated by synaptic outputs from the small percentage of VGLUT2-expressing cones. Several lines of evidence suggest that this wavelet is not caused by synaptic output from VGLUT2expressing cones. First, the wavelet has a similar appearance to a hyperpolarizationactivated current, $I_{\mathrm{h}}$, present in photoreceptor inner segments (Kang Derwent and Linsenmeier, 2001). Second, the time to peak of this wavelet is much faster than that of b-waves. Third, we do not see any wavelet in photopic recording conditions, which would be expected if this wavelet were mediated by VGLUT2-expressing cones. We therefore conclude that any visual signaling mediated by VGLUT2expressing cones was below our detection limits for the ERG recordings. Given that bipolar cells exclusively express VGLUT1, any activation of these cells by VGLUT2-containing cones would not be seen in the MEA recordings.

Intrinsic visual functions are preserved in VGLUT1-null mice Intrinsic visual functions such as light-evoked pupil constriction and photoentrainment are preserved in mice lacking functional photoreceptors caused by inherited retinal degeneration (Freedman et al., 1999; Lucas et al., 2001). Melanopsin-containing
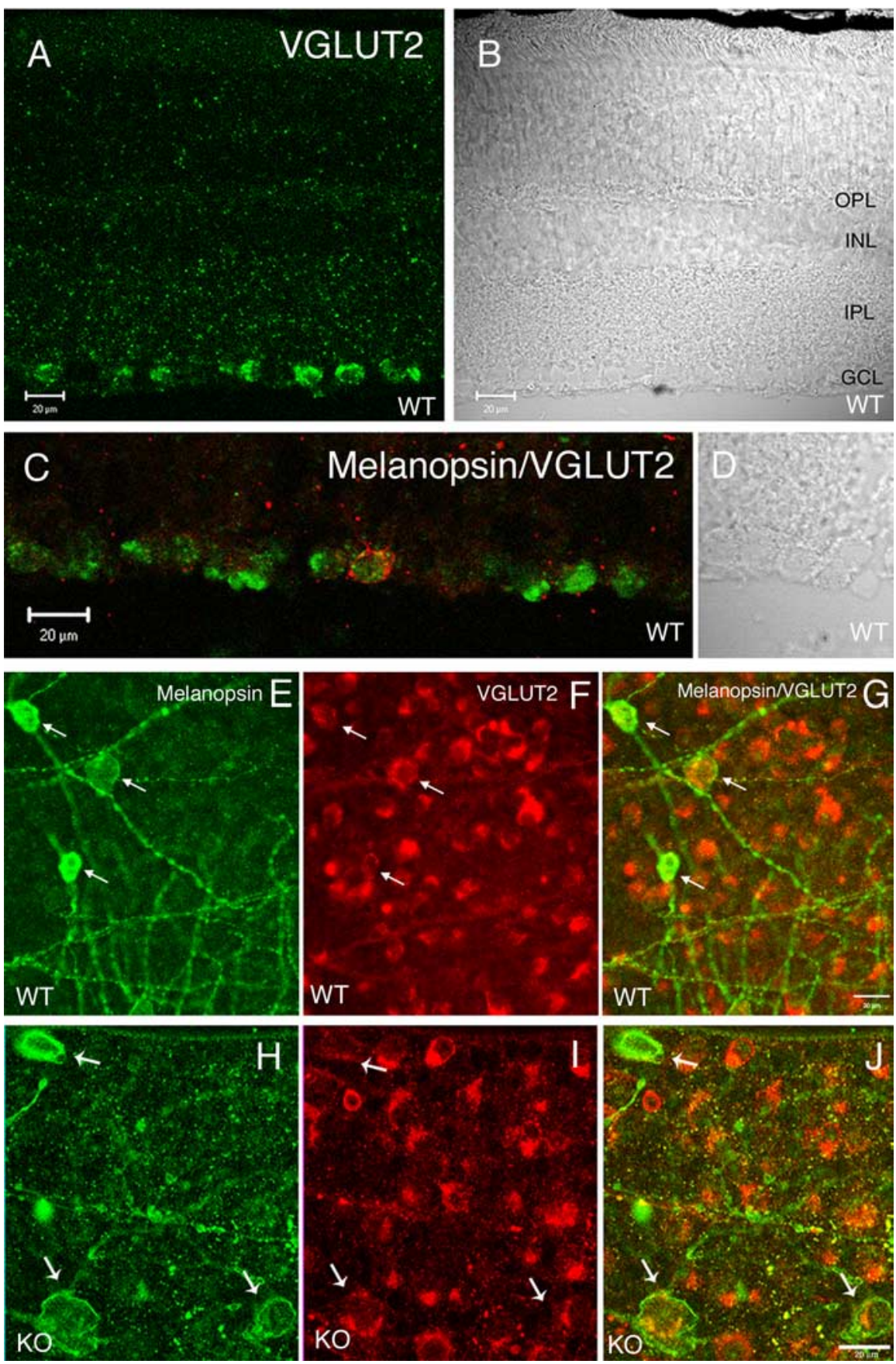

Figure 7. Melanopsin-containing ganglion cells express VGLUT2. A, VGLUT2 mRNA (green) is expressed in cell bodies in the ganglion cell layer in adult WT mouse retina. $\boldsymbol{B}$, Nomarski image of the same retina. OPL, Outer plexiform layer; INL, inner nuclear layer; IPL, inner plexiform layer. C, VGLUT2 mRNA (green) colocalizes with melanopsin immunoreactivity (red) in the ganglion cell layer in WT mouse retina. $\boldsymbol{D}$, Nomarski image of the same retina. $\boldsymbol{E}-\boldsymbol{I}$, Double labeling of whole-mount WT and VGLUT1-null retina immunostained for melanopsin ( $\boldsymbol{E}, \boldsymbol{H}$; green) and VGLUT2 (F, $\boldsymbol{I}$; red). $\boldsymbol{G}, \boldsymbol{J}$, The merged image shows that melanopsin-positive cells express VGLUT2. $\boldsymbol{E}-\boldsymbol{J}$, The arrows indicate cells that are colabeled for melanopsin and VGLUT2. Scale bars, $20 \mu \mathrm{m}$.

ipRGCs are thought to provide the visual signals that mediate this intrinsic vision (Berson et al., 2002; Tu et al., 2005). In VGLUT1null mice, we find light-evoked ipRGCs (Fig. 4A, B), light-evoked pupil constrictions, and photoentrainment (Figs. 5, 6). Given the lack of any detectable signaling from photoreceptors and the anticipated lack of bipolar signaling, our findings provide strong, independent confirmation that light-evoked activity in ipRGCs is sufficient to regulate intrinsic visual functions.

Previous experiments have found that there is labeling for VGLUT1 mRNA in the SCN, whereas there is no detectable 

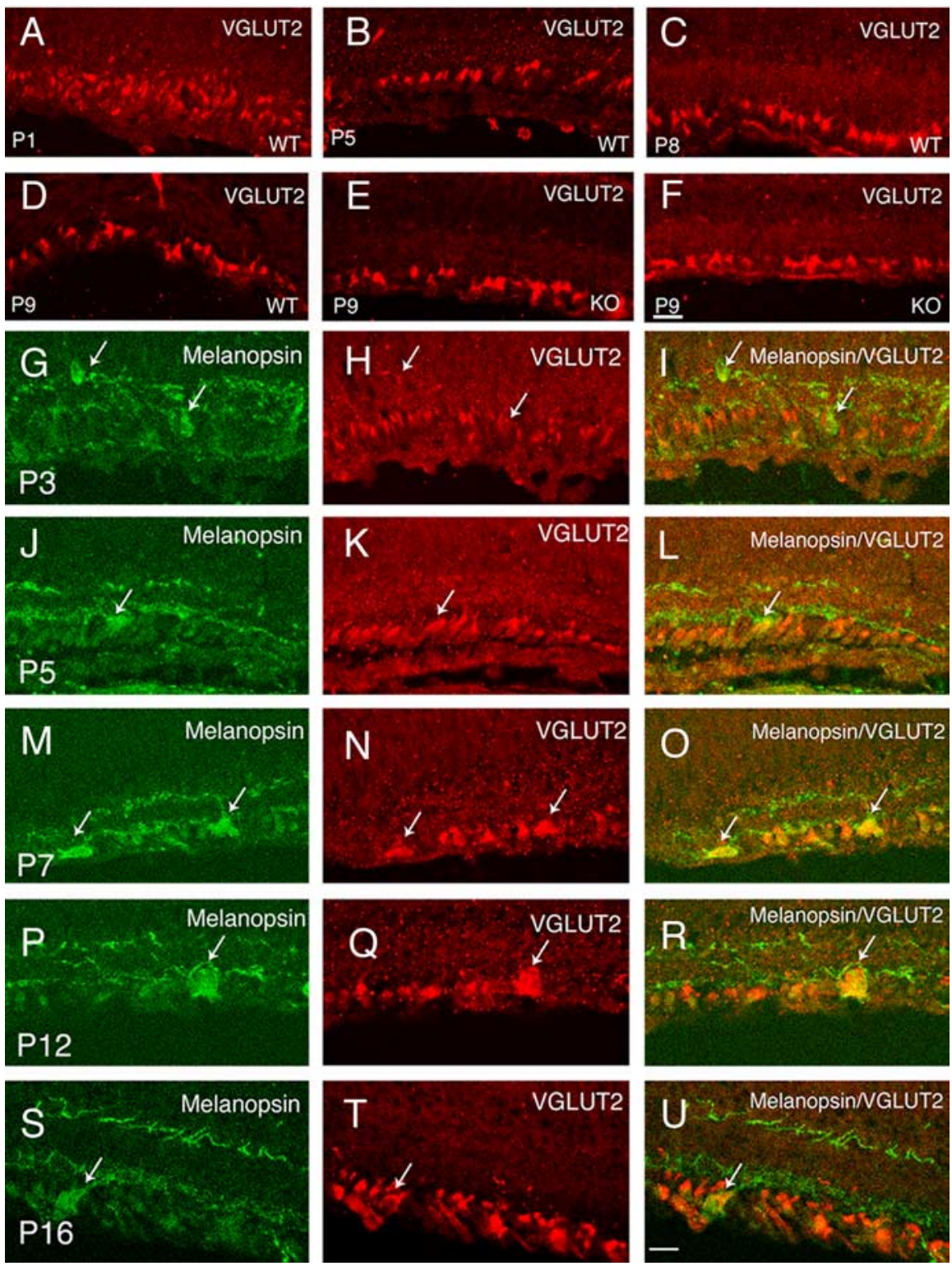

Figure 8. VGLUT2 is expressed in young postnatal retina and colocalizes with melanopsin. $\boldsymbol{A}-\boldsymbol{F}$, VGLUT2 immunostaining (red) strongly labeled cell bodies in P1, P5, P8, and P9 retina. D-F, VGLUT2 immunostaining in P9 retina was indistinguishable between WT $(\boldsymbol{D})$ and VGLUT1-null $(\boldsymbol{E}, \boldsymbol{F})$ retina. $\mathbf{G}-\boldsymbol{U}$, Melanopsin colocalizes with VGLUT2 in young postnatal WT retina. Double-labeling experiments were performed for melanopsin (green) and VGLUT2 (red) in P3, P5, P7, P12, and P16 WT retina. Melanopsin expression colocalized with a subset of VGLUT2 immunostained cell bodies. The arrows indicate cells that are colabeled for melanopsin and VGLUT2. Scale bars, $20 \mu \mathrm{m}$.

VGLUT2 mRNA or immunostaining of cell bodies in the SCN (Ziegler et al., 2002; Lin et al., 2003). These studies predict that even if the retina is able to signal a change in light via VGLUT2mediated RGCs, the transmission of this signaling could be disrupted within and out of the SCN because of a lack of VGLUT1 expression in the VGLUT1-null mouse (Ziegler et al., 2002; Lin et al., 2003). It is therefore surprising that the VGLUT1-null mice are able to be photoentrained.

VGLUT1-mediated visual functions are separable from those of VGLUT2 and VGLUT3

Only three VGLUT isoforms have been identified in the mammalian nervous system (Fremeau et al., 2001). Although VGLUT1 is clearly required for transmitting photoreceptorderived visual signals that provide image-forming signals for the brain, the results from the VGLUT1-null mice show that the glutamatergic neuronal pathways that drive intrinsic non-imageforming functions must use VGLUT2 or VGLUT3. To our knowledge, the present study is the first to demonstrate that melanopsin-containing ipRGCs also express VGLUT2. Although VGLUT3 antibodies immunostain a subset of cat RGCs (Fyk-Kolodziej et al., 2004), we did not detect VGLUT3 immunoreactivity in mouse RGCs. However, we cannot rule out that there are low expression levels of VGLUT3 in the mouse RGCs or that expression of VGLUT3 is only in the terminals of the RGCs and, therefore, not present in the retina. Our results are consistent with the idea that VGLUT2-expressing neurons are conducting the vast majority of visual responses to the SCN and optic pretectal nucleus to mediate photoentrainment and pupil constriction, respectively.

\section{VGLUT2-expressing ipRGCs likely mediate intrinsic visual functions in neonatal animals before functional photoreceptor signaling}

At birth and during early neonatal maturation, before photoreceptors are functional, light induces activity in ipRGCs (Sekaran et al., 2005). Furthermore, at these ages, ipRGCs have already sent axonal projections to the SCN, and light stimulation of the retina can elicit gene expression of c-fos in the SCN as early as P0 (Hannibal and Fahrenkrug, 2004; Sekaran et al., 2005). VGLUT2 expression is present in the GCL at birth (Sherry et al., 2003). In contrast, VGLUT1 and VGLUT3 are not expressed in retina until approximately P7 (Johnson et al., 2003, 2004; Sherry et al., 2003). Therefore, VGLUT2expressing neuronal pathways allow visual inputs to the brain at an age well before photoreceptors are functional. Although little is known about how light controls any behavioral or neuronal functions in neonatal rodents, it is known that the biological clock of premature primate infants is responsive to light (Hao and Rivkees, 1999).

Our multielectrode array experiments indicated that ipRGCs are abundant in young WT and VGLUT1-null mice and then become less abundant with age. Previous studies have found plentiful expression of melanopsin in young postnatal retina and a dramatic decrease after eye opening, suggesting downregulation of melanopsin at a time when photoreceptor-mediated vision becomes functional (Sekaran et al., 2005). We found that abundant ipRGC responses persisted in VGLUT1-null mice to a later age than was observed in WT (see Fig. 4A, bottom). For example, at age P16, ipRGC responses in WT are scarce but were plentiful in VGLUT1-null mice. Nonetheless, by 1 month after birth, the VGLUT1-null mice had a reduction in recorded ipRGCs, as would be expected in WT (Sekaran et al., 2005), indi- 
cating that VGLUT1-null mice have a delay in the developmental decrease of melanopsin.

\section{VGLUT1-null mice as a murine model}

Mutations that cause disruption in photoreceptor signaling often result in the rearrangement of the processes of second-order neurons (Dick et al., 2003; Haeseleer et al., 2004; Chang et al., 2006). For example, in mice that are deficient for the presynaptic cytomatrix protein Bassoon, there is rearrangement of rod bipolar and horizontal dendrites, which is thought to be caused by a lack of photoreceptor-driven synaptic input (Dick et al., 2003). Based on these experiments, which show that photoreceptor input is necessary for the maintenance of second-order processes, our prediction is that there will be disruption of second-order neuronal processes in the VGLUT1-null mouse, which is consistent with preliminary data (data not shown).

The phenotype of the VGLUT1 null is a clear example of complete deletion of functionality in the retina. Many knock-outs of synaptic molecules in the retina result in only partial loss of visual function (Dick et al., 2003; Chang et al., 2006; Duncan et al., 2006). VGLUT1-null mice could serve as a model to test development and function of not only the retina, but of the entire visual system. For example, is vesicular glutamate signaling required for eye-specific segregation inputs in the LGN or for lightdependent pruning of ganglion cell dendrites? Is VGLUT1 expression necessary for the generation of glutamatergic retinal waves? Our results establish that the VGLUT1-null mice are an unambiguous model to address these and other compelling questions.

In conclusion, VGLUT1 expression is essential for photoreceptor-driven signaling. Intrinsic visual functions, such as ipRGC responses, pupil constriction, and photoentrainment, can be signaled via VGLUT2 and do not require VGLUT1 expression anywhere in the pathway.

\section{References}

Barth DS, Goldberg N, Brett B, Di S (1995) The spatiotemporal organization of auditory, visual, and auditory-visual evoked potentials in rat cortex. Brain Res 678:177-190.

Bellocchio EE, Hu H, Pohorille A, Chan J, Pickel VM, Edwards RH (1998) The localization of the brain-specific inorganic phosphate transporter suggests a specific presynaptic role in glutamatergic transmission. J Neurosci 18:8648-8659.

Bellocchio EE, Reimer RJ, Fremeau Jr RT, Edwards RH (2000) Uptake of glutamate into synaptic vesicles by an inorganic phosphate transporter. Science 289:957-960.

Berson DM, Dunn FA, Takao M (2002) Phototransduction by retinal ganglion cells that set the circadian clock. Science 295:1070-1073.

Chang B, Heckenlively JR, Bayley PR, Brecha NC, Davisson MT, Hawes NL, Hirano AA, Hurd RE, Ikeda A, Johnson BA, McCall MA, Morgans CW, Nusinowitz S, Peachey NS, Rice DS, Vessey KA, Gregg RG. (2006) The nob2 mouse, a null mutation in Cacnalf: anatomical and functional abnormalities in the outer retina and their consequences on ganglion cell visual responses. Vis Neurosci 23:11-24.

Copenhagen DR, Jahr CE (1989) Release of endogenous excitatory amino acids from turtle photoreceptors. Nature 341:536-539.

Copenhagen DR, Hemila S, Reuter T (1990) Signal transmission through the dark-adapted retina of the toad (Bufo marinus). Gain, convergence, and signal/noise. J Gen Physiol 95:717-732.

Dick O, tom Dieck S, Altrock WD, Ammermuller J, Weiler R, Garner CC, Gundelfinger ED, Brandstatter JH (2003) The presynaptic active zone protein bassoon is essential for photoreceptor ribbon synapse formation in the retina. Neuron 37:775-786.

Duncan JL, LaVail MM, Yasumura D, Matthes MT, Yang H, Trautmann N, Chappelow AV, Feng W, Earp HS, Matsushima GK, Vollrath D (2003) An RCS-like retinal dystrophy phenotype in mer knockout mice. Invest Ophthalmol Vis Sci 44:826-838.
Duncan JL, Yang H, Doan T, Silverstein RS, Murphy GJ, Nune G, Liu X, Copenhagen D, Tempel BL, Rieke F, Krizaj D (2006) Scotopic visual signaling in the mouse retina is modulated by high-affinity plasma membrane calcium extrusion. J Neurosci 26:7201-7211.

Fisher LJ (1979) Development of synaptic arrays in the inner plexiform layer of neonatal mouse retina. J Comp Neurol 187:359-372.

Freedman MS, Lucas RJ, Soni B, von Schantz M, Munoz M, David-Gray Z, Foster R (1999) Regulation of mammalian circadian behavior by nonrod, non-cone, ocular photoreceptors. Science 284:502-504.

Fremeau Jr RT, Troyer MD, Pahner I, Nygaard GO, Tran CH, Reimer RJ, Bellocchio EE, Fortin D, Storm-Mathisen J, Edwards RH (2001) The expression of vesicular glutamate transporters defines two classes of excitatory synapse. Neuron 31:247-260.

Fremeau Jr RT, Voglmaier S, Seal RP, Edwards RH (2004a) VGLUTs define subsets of excitatory neurons and suggest novel roles for glutamate. Trends Neurosci 27:98-103.

Fremeau Jr RT, Kam K, Qureshi T, Johnson J, Copenhagen DR, StormMathisen J, Chaudhry FA, Nicoll RA, Edwards RH (2004b) Vesicular glutamate transporters 1 and 2 target to functionally distinct synaptic release sites. Science 304:1815-1819.

Fujiyama F, Hioki H, Tomioka R, Taki K, Tamamaki N, Nomura S, Okamoto K, Kaneko T (2003) Changes of immunocytochemical localization of vesicular glutamate transporters in the rat visual system after the retinofugal denervation. J Comp Neurol 465:234-249.

Fyk-Kolodziej B, Dzhagaryan A, Qin P, Pourcho RG (2004) Localization of three vesicular glutamate transporters in the cat retina. J Comp Neurol 475:518-530.

Haeseleer F, Imanishi Y, Maeda T, Possin DE, Maeda A, Lee A, Rieke F, Palczewski K (2004) Free in PMC Essential role of Ca2+-binding protein 4, a Cav1.4 channel regulator, in photoreceptor synaptic function. Nat Neurosci 7:1079-1087.

Hannibal J, Fahrenkrug J (2004) Melanopsin containing retinal ganglion cells are light responsive from birth. NeuroReport 15:2317-2320.

Hao H, Rivkees SA (1999) The biological clock of very premature primate infants is responsive to light. Proc Natl Acad Sci USA 96:2426-2429.

Hattar S, Liao HW, Takao M, Berson DM, Yau KW (2002) Melanopsincontaining retinal ganglion cells: architecture, projections, and intrinsic photosensitivity. Science 295:1065-1070.

Haverkamp S, Wässle H (2004) Characterization of an amacrine cell type of the mammalian retina immunoreactive for vesicular glutamate transporter 3. J Comp Neurol 468:251-263.

Johnson J, Tian N, Caywood MS, Reimer RJ, Edwards RH, Copenhagen DR (2003) Vesicular neurotransmitter transporter expression in developing postnatal rodent retina: GABA and glycine precede glutamate. J Neurosci 23:518-529.

Johnson J, Sherry DM, Liu X, Fremeau Jr RT, Seal RP, Edwards RH, Copenhagen DR (2004) Vesicular glutamate transporter 3 expression identifies glutamatergic amacrine cells in the rodent retina. J Comp Neurol 477:386-398.

Kang Derwent JJ, Linsenmeier RA (2001) Intraretinal analysis of the a-wave of the electroretinogram (ERG) in dark-adapted intact cat retina. Vis Neurosci 18:353-363.

Land PW, Kyonka E, Shamalla-Hannah L (2004) Vesicular glutamate transporters in the lateral geniculate nucleus: expression of VGLUT2 by retinal terminals. Brain Res 996:251-254.

Lin W, McKinney K, Liu L, Lakhlani S, Jennes L (2003) Distribution of vesicular glutamate transporter-2 messenger ribonucleic Acid and protein in the septum-hypothalamus of the rat. Endocrinology 144:662-670.

Liu X, Green CB (2002) Circadian regulation of nocturnin transcription by phosphorylated CREB in Xenopus retinal photoreceptor cells. Mol Cell Biol 22:7501-7511.

Lucas RJ, Douglas RH, Foster RG (2001) Characterization of an ocular photopigment capable of driving pupillary constriction in mice. Nat Neurosci 4:621-626.

Peachey NS, Goto Y, al-Ubaidi MR, Naash MI (1993) Properties of the mouse cone-mediated electroretinogram during light adaptation Neurosci Lett 162:9-11.

Peachey NS, Roveri L, Messing A, McCall MA (1997) Functional consequences of oncogene-induced horizontal cell degeneration in the retinas of transgenic mice. Vis Neurosci 14:627-632.

Ralph RJ, Caine SB (2005) Dopamine D1 and D2 agonist effects on prepulse inhibition and locomotion: comparison of Sprague-Dawley rats to Swiss- 
Webster, 129X1/SvJ, C57BL/6J, and DBA/2J mice. J Pharmacol Exp Ther 312:733-741.

Rentería RC, Tian N, Cang J, Nakanishi S, Stryker MP, Copenhagen DR (2006) Intrinsic ON responses of the retinal OFF pathway are suppressed by the ON pathway. J Neurosci 26:11857-11869.

Robson JG, Frishman LJ (1995) Response linearity and kinetics of the cat retina: the bipolar cell component of the dark-adapted electroretinogram. Vis Neurosci 12:837-850.

Schwartz EA (1986) Synaptic transmission in amphibian retinae during conditions unfavourable for calcium entry into presynaptic terminals. J Physiol (Lond) 376:411-428.

Sekaran S, Lupi D, Jones SL, Sheely CJ, Hattar S, Yau KW, Lucas RJ, Foster RG, Hankins MW (2005) Melanopsin-dependent photoreception provides earliest light detection in the mammalian retina. Curr Biol 15:1099-1107.

Sherry DM, Wang MM, Bates J, Frishman LJ (2003) Expression of vesicular glutamate transporter 1 in the mouse retina reveals temporal ordering in development of rod vs. cone and ON vs. OFF circuits. J Comp Neurol 465:480-498.
Takamori S, Rhee JS, Rosenmund C, Jahn R (2000) Identification of a vesicular glutamate transporter that defines a glutamatergic phenotype in neurons. Nature 407:189-194.

Tian N, Copenhagen DR (2003) Visual stimulation is required for refinement of ON and OFF pathways in postnatal retina. Neuron 39:85-96

Tu DC, Zhang D, Demas J, Slutsky EB, Provencio I, Holy TE, Van Gelder RN (2005) Physiologic diversity and development of intrinsically photosensitive retinal ganglion cells. Neuron 48:987-999.

Van Gelder RN (2003) Making (a) sense of non-visual ocular photoreception. Trends Neurosci 26:458-461.

Wässle H, Regus-Leidig H, Haverkamp S (2006) Expression of the vesicular glutamate transporter vGluT2 in a subset of cones of the mouse retina. J Comp Neurol 496:544-555.

Ziegler DR, Cullinan WE, Herman JP (2002) Distribution of vesicular glutamate transporter mRNA in rat hypothalamus. J Comp Neurol 448:217229. 